\title{
Finding Optimally Balanced Words for Production Planning and Maintenance Scheduling
}

\author{
Jeffrey W. Herrmann \\ A. James Clark School of Engineering \\ University of Maryland \\ College Park, MD 20742 \\ jwh2@umd.edu
}

\section{Abstract}

Balanced words are useful for scheduling mixed-model, just-in-time assembly lines, planning preventive maintenance, managing inventory, and controlling asynchronous transfer mode (ATM) networks. This paper considers the challenging problem of finding a balanced word (a periodic sequence) for a finite set of letters, when the desired densities of the letters in the alphabet are given. Two different measures of balance are considered. This paper presents a branch-and-bound approach for finding optimally balanced words and presents the results of computational experiments to show how problem characteristics affect the time required to find an optimal solution. The optimal solutions are also used to evaluate the performance of an aggregation approach that combines letters with the same density, constructs a word for the aggregated alphabet, and then disaggregates this word into a feasible word for the original alphabet. Computational experiments show that using aggregation with the heuristics not only finds more balanced words but also reduces computational effort for larger instances. Supplementary materials are available for this article. Go to the publisher's online edition of IIE Transactions for proofs, the pseudocode for the algorithms, and additional examples.

Keywords: balanced words, fair sequences, aggregation, cyclic scheduling

\section{Introduction}

Balanced words can be good solutions for problems of finding a fair sequence that allocates capacity to competing demands in such a way that each demand receives, over any time period, a share of the capacity that is approximately proportional to its priority. The idea of fair sequences occurs in many different areas, including scheduling mixed-model, just-in-time assembly lines, planning preventive 
maintenance, inventory management, and controlling asynchronous transfer mode (ATM) networks. Kubiak (2004) provides a good overview of the need for fair sequences in different domains and discusses results for multiple related problems, including the product rate variation problem, generalized pinwheel scheduling, the hard real-time periodic scheduling problem, the periodic maintenance scheduling problem, stride scheduling, minimizing response time variability (RTV), and peer-to-peer fair scheduling.

The routing of jobs in stochastic systems also requires fair sequences. Hajek (1985) considered a queueing system where the interarrival times are independent, identically distributed random variables with finite mean and the server has exponentially distributed processing times. A given fraction of the arriving jobs must be sent to the server, while the rest are sent elsewhere. Hajek showed that a regular admission sequence minimizes the server's expected queue size and the expected waiting time of the admitted jobs. Altman et al. (2000) show that, for very general stochastic systems, the optimal routing of jobs to servers is a balanced sequence. Sano et al. (2004) introduced a generalization of balanced words and showed that using these policies to route jobs minimizes the maximum waiting time.

The remainder of the paper will formulate the balanced word problem, present a branch-andbound approach, discuss heuristics for generating balanced words, and present the aggregation approach. It then discusses the results of computational experiments designed to evaluate the branch-and-bound algorithm and the effectiveness of using aggregation in combination with the different heuristics before concluding the paper.

\section{Balanced Words}

We are given a finite alphabet and a set of densities for the letters in the alphabet. In scheduling problems, these letters correspond to different types of products that need to be produced at different rates. In job routing problems, the letters correspond to different servers that process jobs at different rates. In preventive maintenance problems, the letters correspond to different machines that need service at different rates. The goal is to construct an infinite sequence (word) over the letters in this alphabet in which each letter occurs at a rate that equals the given density. If the densities are all rational, it is 
sufficient to construct a cyclic sequence in which each letter occurs the correct number of times in the finite cycle.

Given a density $p$ in $(0,1)$ and a phase $\theta$ in $[0,1)$, the regular sequence $\sigma(p, \theta)$ has the value $\lfloor(j+1) p+\theta\rfloor-\lfloor j p+\theta\rfloor$ in position $j$. For example, $\sigma\left(\frac{2}{7}, 0\right)=(0001001)^{\infty}$.

For any letter $a$ in the alphabet and any sequence $S$, the indicator sequence $I(s, a)$ has a 1 in position $j$ if $S$ has the letter $a$ in position $j$. Otherwise, $I(s, a)$ has a 0 in position $j$.

The regular word problem is to find a sequence $S$ so that all of the indicator sequences are regular sequences. The complexity of the problem is open (Kubiak, 2009). If the alphabet has at most two distinct densities, then a regular word exists (Altman et al., 2000).

Balanced words are a more general concept than regular words. Two different measures for the degree of balance have been proposed.

Kubiak (2009) gives the following definition: given a finite alphabet $\{1, \ldots, n\}$, a $c$-balanced word is an infinite sequence $U$ such that each position in $U$ is in the alphabet and, if $x$ and $y$ are two factors (subsequences) of the same size, then $\left.|| x\right|_{i}-|y|_{i} \mid \leq c$, where $|x|_{i}$ is the number of times that $i$ occurs in the factor $x$. We will define the count balance of a word $U$ as the minimal such value of $c$. For example, the count balance of the word $(1231211321)^{\infty}$ equals 2 because $|11|_{1}-|23|_{1}=2$ and $\left.|| x\right|_{i}-|y|_{i} \mid \leq 2$ for all factors $x$ and $y$ and all $i$.

If the alphabet has at least two letters, the count balance of a regular word equals 1 . For any given set of densities, the minimal possible count balance is less than or equal to 3 (Kubiak, 2009).

Sano et al. (2004) gives the following definition for a different measure: given a finite alphabet $\{1, \ldots, n\}$, a word $U$ over this alphabet, and a nonnegative integer $m$, a letter $a$ is $m$-balanced in $U$ if, whenever there exists an a-chain $a W a$ in $U$, any factor $W^{\prime}$ in $U$ such that $\left|W^{\prime}\right|=|W|+m+1$ satisfies $\left|W^{\prime}\right|_{a} \geq|W|_{a}+1$. The sequence $U$ is $\mathrm{m}$-balanced if each letter in the alphabet is m-balanced. We will 
define the gap balance of a word $U$ as the minimal such value of $m$. For example, in the infinite sequence $(313132)^{\infty}$, the gap balance of the letters 2 and 3 equals 0 , and the gap balance of the letter 1 equals 2 . Note that the factor 3 in the 1-chain 131 is 2 letters shorter than 323, the longest factor with no instance of the letter 1 . Therefore, the gap balance of this word equals 2 .

The gap balance of a word equals 0 if and only if it is a constant gap word, and the gap balance of a word equals 1 if and only if it is a regular word (Sano et al., 2004). Moreover, the count balance of any word that has a positive gap balance is not larger than the gap balance of that word (Sano et al., 2004).

In other words, the count balance is the maximum difference in the number of copies of a letter for two factors of the same length. The gap balance is the maximum difference in length of two factors that contain the same number of copies of a letter. For both measures, a smaller value implies that the occurrences of each letter are distributed more evenly, which is the goal in many applications. The pseudocode for pseudo-polynomial algorithms that measure the count balance and gap balance of a word are included in the Online Supplement.

Sano et al. (2004) present a search algorithm that randomly generates words and keeps the most balanced one. Otherwise, we know of no algorithms designed specifically to generate balanced words. This paper presents a branch-and-bound algorithm to generate optimally balanced words and heuristics originally used for other, similar problems.

\section{Problem Formulation}

Let $A$ be a finite set of letters $\{1, \ldots, n\}$. Let $U$ be an infinite word over this set such that $U_{t} \in A$

for all integers $t$. The density of letter $a \in A$ is $p_{a}$ if the following limit exists:

$$
p_{a}=\lim _{n \rightarrow \infty} \frac{U_{[0, n)}}{n}
$$

If all of these limits exist, then the sum of the densities must equal 1 :

$$
\sum_{a \in A} p_{a}=1
$$


We assume that all of the densities are rational. Therefore, we consider infinite words $U$ that are the infinite repetition of a finite word $S$; that is, $U=S^{\infty}$. Given an alphabet $A$ and a set of rational densities, there exists a positive integer $T$ and positive integers $x_{1}, \ldots, x_{n}$ such that $p_{i}=x_{i} / T$ for $i=$ $1, \ldots, n$ and $\operatorname{gcd}\left(x_{1}, \ldots, x_{n}\right)=1$. Thus, $x_{1}+\cdots+x_{n}=T$. Hereafter, we will describe an instance by the values of $\left(x_{1}, \ldots, x_{n}\right)$, with $x_{1} \geq x_{2} \geq \cdots \geq x_{n}$.

We will study two versions of the balanced word problem (BWP). They differ only in the measure used. BWP-count uses the count balance measure, and BWP-gap uses the gap balance measure.

Thus, we can describe BWP-count (and BWP-gap) as follows: Given an instance $\left(x_{1}, \ldots, x_{n}\right)$, find a finite word $S$ of length $T$ that minimizes the count balance (gap balance) of the infinite word $U$ that is the infinite repetition of $S$ subject to the constraints that exactly one letter is assigned to each position of $S$ and letter $i$ occurs exactly $x_{i}$ times in $S$ for $i=1, \ldots, n$.

The complexity of BWP-count appears to be open. Given an instance, finding a word with a count balance that equals 1 requires finding a regular word. The complexity of this problem is open (Kubiak, 2009). Likewise, the complexity of BWP-gap appears to be open. Given an instance, finding a word with an gap balance that equals 0 requires finding a constant gap word for $\left(x_{1}, \ldots, x_{n}\right)$. The complexity of the constant gap problem is open (Kubiak, 2004). Nevertheless, these problems are related to the Periodic Maintenance Scheduling Problem, which is NP-complete in the strong sense (Kubiak, 2009), and the RTV problem, which is NP-hard (Corominas et al., 2007).

Consider, as an example, the problem of collecting waste from three different locations in a facility. The locations generate waste at different rates, and the facility manager wishes to schedule waste collection operations so that locations that generate more waste are served (visited) more often (only one location can be served in a time period). Ideally, to prevent excess waste from accumulating, the waste collection operations at any location would be spaced so that the time between them is the same. Suppose 
that the first location generates $4 / 9$ of the total waste, the second generates $1 / 3$ of the total, and the third generates $2 / 9$ of the total. Thus, the demand densities $\left(p_{1}, p_{2}, p_{3}\right)=(4 / 9,1 / 3,2 / 9)$.

This forms the following three-letter instance: $\left(x_{1}, x_{2}, x_{3}\right)=(4,3,2)$, with $T=9$. Consider the word $U=(112231123)^{\infty}$. The count balance of $U$ equals 2, and the gap balance of $U$ equals 3 (because the gap balance of the letter 1 equals 3$)$. Now, consider the word $V=(121312123)^{\infty}$. The count balance

of $V$ also equals 2 (because $|212|_{2}-|131|_{2}=2$ ), but the gap balance of $V$ equals 2 (because the gap balance of the letter 2 equals 2 ).

In terms of our waste collection example, in both sequences each location receives a number of visits that is proportional to its demand (the amount of waste generated). Location 1 , for instance, is served during 4 of every 9 time periods. The second sequence, however, more evenly distributes the operations among the three locations, so less waste will accumulate, and each visit to a location will collect approximately the same amount of waste.

\section{Special Cases}

Two special cases are easy to solve. If there is only one product, then there is only one feasible word, and both its gap balance and its count balance equal zero.

If $n>1$ and all of the $x_{i}$ are equal, then an optimal balanced word has $x_{1}$ copies of a permutation of $\{1, \ldots, n\}$. An optimal word's count balance equals 1 , and its gap balance equals 0 .

\section{Branch-and-Bound}

Branch-and-bound is a well-established approach for finding optimal solutions to scheduling problems. To solve BWP-gap and BWP-count, we developed a branch-and-bound approach that uses the same branching strategy to solve both problems. Naturally, the two problems require different lower bounds. The following paragraphs describe the branching strategy; see also the flowchart in Figure 1.

The approach creates an initial node with no letters assigned to any position. At this initial node, because the problem involves a cycle, the approach assigns the first copy of letter 1 to the first position in the word. Letter 1 is the active letter. 
The next copy of the active letter is assigned to the next open position in the word. If this is the last copy of the active letter, then the algorithm creates a new node with this partial word. After processing this node, the algorithm tries the next open position for the current (last) copy of the active letter. That continues until there are no remaining open positions to try, at which point the algorithm moves back to the previous copy of the active letter.

If this is not the last copy of the active letter and it is not the first or second copy of the active letter, then there are at least two consecutive gaps, and the algorithm computes a lower bound based on this incomplete set of gaps. If the lower bound is less than the current upper bound, then the algorithm moves on to place the next copy of the active letter. Else, it tries the next open position for the current copy of the active letter. That continues until there are no remaining open positions to try (note that enough open positions must remain for the remaining copies of the active letter).

At the new node, the algorithm calculates the balance of the last letter placed. If the lower bound is greater than the current upper bound, it prunes the node. If this letter is not the last letter in the alphabet and the next letter requires more than one copy, then the algorithm selects the next letter as the active letter and begins placing copies of that as described above. 


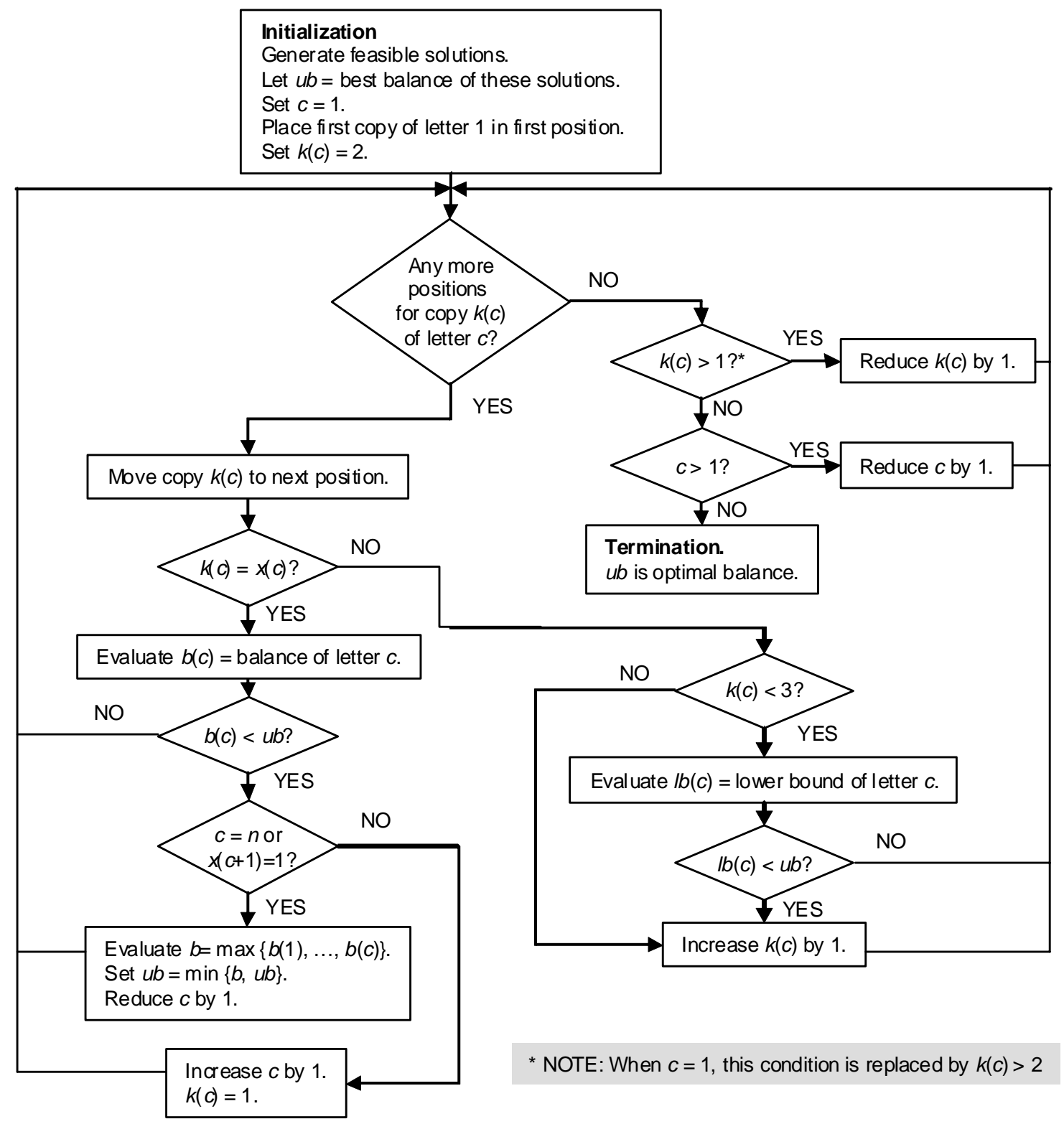

Figure 1. Flowchart for branch-and-bound algorithm.

In this figure $x(c)$ is used to represent $x_{c}$.

If the last letter placed is the last letter in the alphabet or all of the remaining letters have $x_{i}=1$ (in which case placing the remaining letters cannot increase the balance), the algorithm updates the upper bound and returns to the previous node. 
The procedures to calculate the gap balance and count balance lower bounds for an incomplete set of gaps are similar to the procedures to calculate the gap balance and count balance of a complete schedule. They consider only the gaps that are given but do not combine the first and last gaps as if they were consecutive because there are missing gaps of unknown size between them. For instance, suppose that a letter should have five copies in the word, but only four copies have been placed. Thus, there are three known consecutive gaps. There are also two unknown gaps. The balance calculations consider only the three known gaps and the two combinations of two consecutive known gaps. The calculations do not combine the first and last known gaps because they are not contiguous.

\section{Heuristics}

To construct solutions for the BWP-count (and BWP-gap), we consider a number of heuristics that have been proposed for related problems. The following discussion briefly describes the heuristics. Detailed algorithms and examples are given in the Online Supplement. We conducted extensive computational testing to evaluate the performance and computational effort of these heuristics and used these heuristics with the aggregation approach presented later.

GR. The greedy regular (GR) algorithm, presented by van der Laan (2005), tries to make the sequence of each letter resemble a regular sequence as much as possible. The highest-density letter will have a regular sequence. The sequences for the other letter are regular with respect to the sequences of the higher-density letters. The GR algorithm generates a periodic policy. The computational effort of the GR algorithm is $\mathrm{O}(n T)$.

Stride. Waldspurger and Weihl (1995) considered the problem of scheduling multithreaded computer systems. In such a system, there are multiple clients, and each client has a number of tickets. A client with twice as many tickets as another client should be allocated twice as many quanta (time slices) in any given time interval. Waldspurger and Weihl introduced the stride scheduling algorithm to solve this problem. They also presented a hierarchical stride scheduling approach that uses a balanced binary tree to group clients, uses stride scheduling to allocate quanta to the groups, and then, within each group, uses stride scheduling to allocate quanta to the clients. Although they note that grouping clients with the 
same number of tickets would be desirable, their approach does not exploit this. Indeed, the approach does not specify how to create the binary tree. Kubiak (2004) showed that the stride scheduling algorithm is the same as Jefferson's method of apportionment and is an instance of the more general parametric method of apportionment (Balinski and Young, 1982). Thus, the stride scheduling algorithm can be parameterized.

Two of the heuristics are versions of the parameterized stride scheduling algorithm, which builds a fair sequence and performs well at minimizing the maximum absolute deviation (Kubiak, 2004). The algorithm has a single parameter $\delta$ that can range from 0 to 1 . This parameter affects the relative priority of low-density letters and their absolute position within the sequence. When $\delta$ is near 0 , low-density letters will be positioned earlier in the sequence. When $\delta$ is near 1 , low-density letters will be positioned later in the sequence.

We will use the stride scheduling algorithm with $\delta=0.5$ and $\delta=1$ to generate periodic policies. The computational effort of the parameterized stride scheduling algorithm is $\mathrm{O}(n T)$.

Bottleneck. The bottleneck minimization problem (Steiner and Yeomans, 1993) is related to fair sequencing of a mixed-model manufacturing facility. To solve the BWP, we use an algorithm that Steiner and Yeomans (1993) developed. The bottleneck algorithm calculates an earliest and latest start time for each unit of demand (each letter in the alphabet) and then allocates to each position in the word the eligible product (letter) with the smallest latest start time. This heuristic runs in $O(n T)$ time.

Search. Sano et al. (2004) proposed a search algorithm for finding balanced words. The search randomly selects phases $\left\{\phi_{1}, \ldots, \phi_{n}\right\}$ and then uses these phases to construct a word. If the resulting word has a lower gap balance than the best one found so far, it is saved.

To generate a word from a set of phases, the algorithm starts at the first position and selects the letter $i$ with the minimal phase. The algorithm increases $\phi_{i}$ by $T / x_{i}$ and moves to the next position. 


\section{Aggregation}

To improve the performance of these heuristics, we employed an aggregation approach that first aggregates an alphabet, constructs a solution for the aggregated alphabet, and then disaggregates that solution. Aggregation is a well-known and valuable technique for solving optimization problems, especially large-scale mathematical programming problems. Model aggregation replaces a large optimization problem with a smaller, auxiliary problem that is easier to solve (Rogers et al., 1991). The solution to the auxiliary model is then disaggregated to form a solution to the original problem. Model aggregation has been applied to a variety of production and distribution problems, including machine scheduling problems. For example, Rock and Schmidt (1983) and Nowicki and Smutnicki (1989) aggregated the machines in a flow shop scheduling problem to form a two-machine problem.

We previously developed this aggregation scheme, which is similar to the substitution concept discussed by Wei and Liu (1983), to generate solutions for the RTV problem and showed that using aggregation with parameterized stride scheduling and an improvement heuristic generates solutions with lower RTV and reduces the computational effort (Herrmann, 2007, 2009, 2011; see also Herrmann, 2010). This paper builds on the previous work but considers a more general problem.

The aggregation approach used here repeatedly aggregates an alphabet until it cannot be aggregated any more. Each aggregation combines letters that have the same density into a group. These letters are removed, and the group becomes a new letter in the new aggregated alphabet. The aggregation algorithm and an example are available in the Online Supplement.

The aggregation procedure generates a sequence of instances $I_{0}, \ldots, I_{H}$. ( $H$ is the index of the last aggregation created.) The aggregation can be done at most $n-1$ times because the number of letters decreases by at least one each time an aggregation occurs. Thus $H \leq n-1$. Aggregation runs in $\mathrm{O}\left(n^{2}\right)$ time because each aggregation requires $\mathrm{O}(n)$ time and there are at most $n-1$ aggregations. 


\section{Disaggregation}

When aggregation is complete, we must find a feasible solution for the aggregated instance $I_{H}$ and then disaggregate that solution. We will use the heuristics presented earlier to construct $S_{H}$, a feasible solution for the instance $I_{H}$. Disaggregating $S_{H}$ requires $H$ steps that correspond to the aggregations that generated the instances $I_{1}$ to $I_{H}$, but they will, naturally, be considered in reverse order. We disaggregate $S_{H}$ to generate $S_{H-1}$ and then continue to disaggregate each solution in turn to generate $S_{H-2}, \ldots, S_{0} . S_{0}$ is a feasible solution for $I_{0}$, the original instance.

The basic idea of disaggregating a solution $S_{k}$ is to replace each new letter with the letters used to form it. The disaggregation algorithm and an example are available in the Online Supplement.

As noted earlier, there are at most $n-1$ aggregations. Because each solution disaggregation requires $\mathrm{O}(T)$ effort, disaggregation runs in $\mathrm{O}(n T)$ time in total.

\section{Disaggregating Balanced Words}

How does disaggregating a word affect its count balance or its gap balance? That is, is the count balance (or gap balance) of the disaggregated word equal to the count balance of the aggregated word?

Theorem 1. Disaggregating a word that has a positive count balance does not increase its count balance.

(The proof appears in the Online Supplement.)

If the aggregated alphabet has only one letter, then the count balance of the aggregated word equals zero. The initial disaggregation of this word will yield a regular word. This word's count balance will equal 1.

Theorem 2. Disaggregating a word does not increase its gap balance.

(The proof appears in the Online Supplement.) 


\section{Computational Results}

The purpose of the first set of computational experiments was to determine how characteristics of the BWP affect the computational effort of the branch-and-bound algorithm and to evaluate the performance of the heuristics with and without aggregation. All of the algorithms were implemented in Matlab and executed using Matlab R2006b on a Dell Optiplex GX745 with Intel Core2Duo CPU 6600 @ 2.40 GHz and 2.00 GB RAM running Microsoft Windows XP Professional Version 2002 Service Pack 3.

To generate instances, we used the following scheme. First, we set the value of $T$ and the number of letters $n$. Then, for this combination, we generated $T-n$ random numbers from a discrete uniform distribution over $\{1, \ldots, n\}$. We then let $x_{i}$ equal one plus the number of copies of $i$ in the set of $T-n$ random numbers (this avoided the possibility that any $x_{i}=0$ ). For the first set of computational experiments, we generated 100 instances for each of 13 combinations of $T$ and $n$ shown in Table 1. For $n \geq 10$, all of the generated instances can be aggregated. For $n=5$, only some of the instances can be aggregated.

In our implementation of the branch-and-bound, the initial upper bound for an instance was generated by running the bottleneck, GR, and stride scheduling heuristics on that instance. The stride was run twice: the first time with its parameter set to 0.5 and a second time with its parameter set to 1 . The smallest count balance of these four solutions was used an upper bound for the search for an optimal count balance word. The smallest gap balance of these four solutions was used an upper bound for the search for an optimal gap balance word. We used all four heuristics because they run quickly.

Finally, we applied the five heuristics to the original and aggregated instances. For each instance, we constructed solutions as follows. First, we applied one of the basic heuristics to the instance (we call this the $\mathrm{H}$ solution). Next, we aggregated the instance. For the aggregate instance, we applied the heuristic to construct an aggregated solution. We disaggregated this solution to construct the AHD solution. This makes two policies using one basic heuristic. We repeated this for the remaining basic heuristics for a total of 10 policies. Thus, we had a total of 12 solutions for each instance. 
The results (see Tables 2 and 3) show that the gap and count branch-and-bound algorithms required more computational effort to solve instances with medium levels of $n$ (near $T / 2$ ). This occurs because, for each letter considered, there are many more combinations of positions that must be evaluated. This effort increased as $T$ increased. The algorithms required significantly less computational effort to solve instances with high levels of $n$ (near $T$ ) because many letters will need only one position, and the algorithms don't need to consider which particular positions are assigned to these letters. In general, the gap branch-and-bound algorithms required more computational effort than the count branchand-bound algorithm. This is due to the better count balances of the solutions generated by the heuristics. For $61 \%$ (789 of 1300) of the instances, the best count balance of the heuristics' solutions was optimal. On the other hand, the best gap balance of the heuristics' solutions was optimal for only $28 \%$ ( 363 of 1300) of the instances.

Tables 4 through 7 summarize the results of using the heuristics and aggregation. (For problem sets with $n=5$, no results are given for the AHD solutions because not all of the instances could be aggregated.) For the smallest instances $(T=15)$, the search procedure is able to find optimal solutions. The other heuristics do not perform as well. With $T=15$ and $n=5$, aggregation helps the bottleneck, GR, and stride heuristics find more balanced solutions than they would without aggregation. As $T$ increases, the solution quality of the heuristics generally declines (there is a bigger difference between the average optimal balance and the average balance of the heuristics' solutions, and the number of optimal solutions found declines). One exception is the problem set with $T=25$ and $n=20$. These are relatively simple instances in which at least 15 letters have $x_{i}=1$. The search heuristic finds an optimal solution for every instance with or without aggregation. The GR heuristic constructs an optimal solution for every instance without aggregation. The bottleneck and stride heuristics construct no optimal solutions without aggregation but construct an optimal solution for every instance when used with aggregation.

Overall, the search procedure (without aggregation) and the stride procedure (with aggregation) generate more balanced words more often than the other heuristics. The stride algorithm with aggregation 
requires less than 0.0005 seconds per instance on these instances. The search procedure, however, requires 0.4 to 0.8 seconds per instance.

Table 1. Combinations of $T$ and $n$ used to generate instances.

\begin{tabular}{rrrrrrrrrrrrrr}
\hline$T$ & 15 & 15 & 20 & 20 & 20 & 25 & 25 & 25 & 25 & 30 & 30 & 30 & 30 \\
$n$ & 5 & 10 & 5 & 10 & 15 & 5 & 10 & 15 & 20 & 5 & 10 & 15 & 20 \\
\hline
\end{tabular}


Table 2. Distribution and average of the time for the gap balance branch-and-bound to find an optimal solution to the instances. Problem sets are labeled as $T-n$.

\begin{tabular}{|c|c|c|c|c|}
\hline Interval of times (secs) & $15-5$ & $15-10$ & & \\
\hline$(0,0.1)$ & 75 & 100 & & \\
\hline$(0.1,1)$ & 25 & & & \\
\hline$(1,10)$ & & & & \\
\hline$(10,100)$ & & & & \\
\hline$(100,1000)$ & & & & \\
\hline$(1000,3000)$ & & & & \\
\hline Average time (secs) & 0.0854 & 0.0192 & & \\
\hline Interval of times (secs) & $20-5$ & $20-10$ & $20-15$ & \\
\hline$(0,0.1)$ & 1 & 7 & 100 & \\
\hline$(0.1,1)$ & 98 & 81 & & \\
\hline$(1,10)$ & 1 & 9 & & \\
\hline$(10,100)$ & & 3 & & \\
\hline$(100,1000)$ & & & & \\
\hline$(1000,3000)$ & & & & \\
\hline Average time (secs) & 0.5070 & 3.0025 & 0.0226 & \\
\hline Interval of times (secs) & $25-5$ & $25-10$ & $25-15$ & $25-20$ \\
\hline$(0,0.1)$ & & & 16 & 100 \\
\hline$(0.1,1)$ & 2 & 45 & 84 & \\
\hline$(1,10)$ & 72 & 28 & & \\
\hline$(10,100)$ & 26 & 25 & & \\
\hline$(100,1000)$ & & 0 & & \\
\hline$(1000,3000)$ & & 2 & & \\
\hline Average time (secs) & 17.7838 & 65.3033 & 0.1575 & 0.0308 \\
\hline Interval of times (secs) & $30-5$ & $30-10$ & $30-15$ & $30-20$ \\
\hline$(0,0.1)$ & & & & 32 \\
\hline$(0.1,1)$ & 1 & & 41 & 68 \\
\hline$(1,10)$ & 12 & 44 & 25 & \\
\hline$(10,100)$ & 45 & 40 & 16 & \\
\hline$(100,1000)$ & 28 & 14 & 16 & \\
\hline$(1000,3000)$ & 14 & 2 & 2 & \\
\hline Average time (secs) & 387.0133 & 64.9798 & 112.5814 & 0.2163 \\
\hline
\end{tabular}


Table 3. Distribution and average of the time for the count balance branch-and-bound to find an optimal solution to the instances. Problem sets are labeled as $T-n$.

\begin{tabular}{|c|c|c|c|c|}
\hline Interval of times (secs) & $15-5$ & $15-10$ & & \\
\hline$(0,0.1)$ & 88 & 100 & & \\
\hline$(0.1,1)$ & 12 & & & \\
\hline$(1,10)$ & & & & \\
\hline$(10,100)$ & & & & \\
\hline$(100,1000)$ & & & & \\
\hline$(1000,3000)$ & & & & \\
\hline Average time (secs) & 0.0600 & 0.0056 & & \\
\hline Interval of times (secs) & $20-5$ & $20-10$ & $20-15$ & \\
\hline$(0,0.1)$ & 10 & 70 & 100 & \\
\hline$(0.1,1)$ & 90 & 18 & & \\
\hline$(1,10)$ & & 9 & & \\
\hline$(10,100)$ & & 3 & & \\
\hline$(100,1000)$ & & & & \\
\hline$(1000,3000)$ & & & & \\
\hline Average time (secs) & 0.2353 & 1.3903 & 0.0076 & \\
\hline Interval of times (secs) & $25-5$ & $25-10$ & $25-15$ & $25-20$ \\
\hline$(0,0.1)$ & & 20 & 90 & 100 \\
\hline$(0.1,1)$ & 3 & 28 & 10 & \\
\hline$(1,10)$ & 97 & 35 & & \\
\hline$(10,100)$ & & 17 & & \\
\hline$(100,1000)$ & & & & \\
\hline$(1000,3000)$ & & & & \\
\hline Average time (secs) & 1.9915 & 4.2832 & 0.0462 & 0.0092 \\
\hline Interval of times (secs) & $30-5$ & $30-10$ & $30-15$ & $30-20$ \\
\hline$(0,0.1)$ & & & 1 & 90 \\
\hline$(0.1,1)$ & 5 & 12 & 53 & 10 \\
\hline$(1,10)$ & 81 & 80 & 14 & \\
\hline$(10,100)$ & 14 & 8 & 26 & \\
\hline$(100,1000)$ & & & 5 & \\
\hline$(1000,3000)$ & & & 1 & \\
\hline Average time (secs) & 3.8305 & 6.4256 & 32.5523 & 0.0596 \\
\hline
\end{tabular}


Table 4. Average values of the count balance for the H and AHD solutions generated using five basic heuristics and the branch-and-bound.

\begin{tabular}{rrrllllllllll} 
& & & \multicolumn{2}{c}{ Search } & \multicolumn{2}{c}{ Bottleneck } & \multicolumn{2}{c}{ GR } & \multicolumn{2}{c}{ Stride 0.5 } & \multicolumn{2}{c}{ Stride 1.0 } \\
\cline { 3 - 13 }$T$ & $n$ & Opt. & H & AHD & H & AHD & H & AHD & H & AHD & H & AHD \\
\hline 15 & 5 & 1.75 & 1.75 & & 1.96 & & 1.94 & & 2.06 & & 2.14 & \\
& 10 & 1.00 & 1.00 & 1.12 & 2.53 & 1.12 & 1.12 & 1.12 & 2.72 & 1.12 & 2.79 & 1.12 \\
\hline 20 & 5 & 1.88 & 1.88 & & 2.01 & & 2.02 & & 2.15 & & 2.23 & \\
& 10 & 1.09 & 1.39 & 1.34 & 2.21 & 1.23 & 1.90 & 1.41 & 2.24 & 1.23 & 2.76 & 1.39 \\
& 15 & 1.00 & 1.00 & 1.48 & 2.54 & 1.48 & 1.53 & 1.53 & 2.55 & 1.48 & 2.55 & 1.48 \\
\hline 25 & 5 & 1.98 & 1.98 & & 2.00 & & 2.10 & & 2.10 & & 2.21 & \\
& 10 & 1.25 & 1.59 & 1.79 & 2.33 & 1.87 & 2.00 & 1.87 & 2.48 & 1.75 & 2.50 & 1.83 \\
& 15 & 1.00 & 1.11 & 1.27 & 2.80 & 1.45 & 1.76 & 1.73 & 3.02 & 1.20 & 3.39 & 1.28 \\
& 20 & 1.00 & 1.00 & 1.00 & 2.39 & 1.00 & 1.00 & 1.37 & 2.41 & 1.00 & 2.41 & 1.00 \\
\hline 30 & 5 & 1.95 & 1.95 & & 1.99 & & 1.99 & & 2.13 & & 2.19 & \\
& 10 & 1.84 & 1.96 & 1.90 & 2.72 & 1.88 & 2.00 & 1.95 & 2.74 & 1.86 & 2.95 & 1.88 \\
& 15 & 1.11 & 1.98 & 1.79 & 2.57 & 1.80 & 1.98 & 1.88 & 2.24 & 1.79 & 2.88 & 1.87 \\
& 20 & 1.00 & 1.86 & 1.58 & 2.89 & 1.61 & 1.82 & 1.61 & 3.00 & 1.61 & 3.21 & 1.61 \\
\hline
\end{tabular}

Table 5. Average values of the gap balance for the $\mathrm{H}$ and AHD solutions generated using five basic heuristics and the branch-and-bound.

\begin{tabular}{rrrrrrccccccc} 
& & \multicolumn{4}{c}{ Search } & \multicolumn{2}{c}{ Bottleneck } & \multicolumn{2}{c}{ GR } & \multicolumn{2}{c}{ Stride 0.5 } & \multicolumn{2}{c}{ Stride 1.0 } \\
\cline { 3 - 14 }$T$ & $n$ & Opt. & H & AHD & H & AHD & H & AHD & H & AHD & H & AHD \\
\hline 15 & 5 & 1.72 & 1.72 & & 3.24 & & 3.81 & & 3.29 & & 3.57 & \\
& 10 & 1.00 & 1.00 & 1.12 & 6.85 & 1.12 & 1.24 & 1.12 & 7.62 & 1.12 & 7.76 & 1.12 \\
\hline 20 & 5 & 1.87 & 1.87 & & 3.49 & & 5.21 & & 3.43 & & 3.71 & \\
& 10 & 1.01 & 1.31 & 1.28 & 7.10 & 1.30 & 2.89 & 1.71 & 6.77 & 1.15 & 8.19 & 1.37 \\
& 15 & 0.53 & 0.53 & 1.01 & 10.50 & 1.01 & 1.06 & 1.11 & 12.09 & 1.01 & 12.11 & 1.01 \\
\hline 25 & 5 & 2.21 & 2.23 & & 3.62 & & 6.20 & & 3.55 & & 3.78 & \\
& 10 & 1.27 & 1.66 & 2.08 & 7.70 & 2.46 & 5.88 & 3.63 & 6.85 & 2.00 & 8.55 & 2.14 \\
& 15 & 1.00 & 1.11 & 1.31 & 11.03 & 1.87 & 2.62 & 3.01 & 11.96 & 1.25 & 12.92 & 1.43 \\
& 20 & 1.00 & 1.00 & 1.00 & 15.04 & 1.00 & 1.00 & 1.74 & 16.52 & 1.00 & 16.56 & 1.00 \\
\hline 30 & 5 & 2.36 & 2.38 & & 3.80 & & 6.67 & & 3.73 & & 3.85 & \\
& 10 & 1.84 & 1.98 & 2.09 & 7.74 & 3.14 & 8.06 & 5.12 & 7.28 & 2.26 & 8.39 & 2.49 \\
& 15 & 0.82 & 1.98 & 1.71 & 11.37 & 1.79 & 4.10 & 3.52 & 10.23 & 1.69 & 12.75 & 1.87 \\
& 20 & 0.24 & 1.77 & 1.31 & 14.69 & 1.55 & 2.09 & 2.34 & 16.40 & 1.29 & 17.38 & 1.43 \\
\hline
\end{tabular}


Table 6. Number of optimal count balance solutions for the $\mathrm{H}$ and AHD solutions generated using five basic heuristics.

\begin{tabular}{rrrrrrrrrrrr} 
& & \multicolumn{2}{c}{ Search } & \multicolumn{2}{c}{ Bottleneck } & \multicolumn{2}{c}{ GR } & \multicolumn{2}{c}{ Stride 0.5 } & \multicolumn{2}{c}{ Stride 1.0 } \\
\cline { 2 - 12 }$T$ & $n$ & \multicolumn{1}{c}{ H } & AHD & H & AHD & H & AHD & H & AHD & H & AHD \\
\hline 15 & 5 & 100 & & 80 & & 81 & & 73 & & 67 & \\
& 10 & 100 & 88 & 0 & 88 & 88 & 88 & 0 & 88 & 0 & 88 \\
\hline 20 & 5 & 100 & & 87 & & 86 & & 73 & & 65 & \\
& 10 & 70 & 75 & 7 & 86 & 19 & 68 & 3 & 86 & 3 & 70 \\
& 15 & 100 & 52 & 0 & 52 & 47 & 47 & 0 & 52 & 0 & 52 \\
\hline 25 & 5 & 100 & & 98 & & 88 & & 88 & & 78 & \\
& 10 & 66 & 46 & 5 & 38 & 25 & 38 & 2 & 50 & 0 & 42 \\
& 15 & 89 & 73 & 0 & 55 & 24 & 27 & 0 & 80 & 0 & 72 \\
& 20 & 100 & 100 & 0 & 100 & 100 & 63 & 0 & 100 & 0 & 100 \\
\hline 30 & 5 & 100 & & 96 & & 96 & & 82 & & 76 & \\
& 10 & 88 & 94 & 13 & 96 & 84 & 89 & 10 & 98 & 4 & 96 \\
& 15 & 13 & 32 & 0 & 31 & 13 & 23 & 0 & 32 & 0 & 24 \\
& 20 & 14 & 42 & 0 & 39 & 18 & 39 & 0 & 39 & 0 & 39 \\
\hline
\end{tabular}

Table 7. Number of optimal gap balance solutions for the $\mathrm{H}$ and AHD solutions generated using five basic heuristics.

\begin{tabular}{rrrrrrrrrrrrr} 
& & \multicolumn{2}{c}{ Search } & \multicolumn{2}{c}{ Bottleneck } & \multicolumn{2}{c}{ GR } & \multicolumn{2}{c}{ Stride 0.5 } & \multicolumn{2}{c}{ Stride 1.0 } \\
\cline { 2 - 13 }$T$ & $n$ & H & AHD & H & AHD & H & AHD & H & AHD & H & AHD \\
\hline 15 & 5 & 100 & & 6 & & 18 & & 5 & & 3 & \\
& 10 & 100 & 88 & 0 & 88 & 88 & 88 & 0 & 88 & 0 & 88 \\
\hline 20 & 5 & 100 & & 3 & & 10 & & 2 & & 1 & \\
& 10 & 70 & 73 & 0 & 83 & 17 & 60 & 0 & 86 & 0 & 66 \\
& 15 & 100 & 52 & 0 & 52 & 47 & 47 & 0 & 52 & 0 & 52 \\
\hline 25 & 5 & 98 & & 9 & & 7 & & 11 & & 0 & \\
& 10 & 62 & 41 & 0 & 15 & 2 & 13 & 0 & 45 & 0 & 35 \\
& 15 & 89 & 73 & 0 & 55 & 24 & 27 & 0 & 80 & 0 & 72 \\
& 20 & 100 & 100 & 0 & 100 & 100 & 63 & 0 & 100 & 0 & 100 \\
\hline 30 & 5 & 98 & & 4 & & 3 & & 9 & & 3 & \\
& 10 & 86 & 75 & 0 & 38 & 0 & 18 & 0 & 71 & 0 & 51 \\
& 15 & 13 & 31 & 0 & 30 & 4 & 12 & 0 & 32 & 0 & 23 \\
& 20 & 14 & 42 & 0 & 39 & 18 & 39 & 0 & 39 & 0 & 39 \\
\hline
\end{tabular}




\section{Comparing the Heuristics on Larger Instances}

The purpose of the second set of computational experiments was to determine the relative performance of the heuristics with and without aggregation. These instances were too large to solve efficiently using the branch-and-bound algorithms.

We generated 1,800 instances using the same scheme described earlier. We generated 100 instances for each of the combinations of $T$ and $n$ shown in Table 8 .

All of these instances can be aggregated. For each instance, we constructed solutions as follows. First, we applied one of the basic heuristics to the instance (we call this the $\mathrm{H}$ solution). Next, we aggregated the instance. For the aggregate instance, we applied the heuristic to construct an aggregated solution. We disaggregated this solution to construct the AHD solution. This makes two policies using one basic heuristic. We repeated this for the remaining basic heuristics for a total of 10 policies.

Before discussing the results of the heuristics, we consider first how many times that an instance could be aggregated. Table 9 shows that the average number of aggregations almost always decreases as $n$ increases. When $T=100$, the average number of aggregations increases as $n$ increases from 10 to 20 . The average number of aggregations per instance is near six for $T=100$ and $n=20$, but, as $n$ increases, this decreases to just over two.

As $n$ approaches $T$, the average number of letters in the aggregated instances also decreases because the aggregation depends upon the number of distinct values of $x_{i}$. Each distinct value leads to an aggregation of multiple letters and generates a letter in the aggregated instance. Thus, the number of letters in the aggregated instance generally equals the number of aggregations needed to create it. Of course, there are some cases in which two groups can be combined, which increases the number of aggregations and reduces the number of letters, and some letters may have unique values, but this occurred less often as $n$ increased. As $n$ approaches $T$, the number of distinct values decreases, so there are fewer aggregations and fewer letters in the aggregated instances. 
We will first consider the results for minimizing the count balance. As shown in Table 10, the stride scheduling and bottleneck heuristics generated words with larger count balances. The performance of the GR heuristic improved as $n$ increased (and approached T). Using aggregation led to the best solutions with the stride, bottleneck, and search algorithms. Aggregation was not as useful with the GR heuristic.

As shown in Table 11, with the gap balance, the general trend is similar, but the differences are greater because the gap balance can be quite large for some words. The bottleneck heuristic generated words with larger gap balances. Using aggregation with the stride and search heuristics consistently generated the best solutions. The GR heuristic generated poor-quality solutions when $n$ was small, but, as $n$ approached $T$, the solution quality dramatically improved. Interestingly, using aggregation with the GR heuristic generated better solutions when $n$ was small, but constructed more unbalanced solutions as $n$ approached $T$.

We also measured the clock time needed to generate these policies. Table 12 summarizes these results, and Figure 2 shows the average time needed to generate the different policies for different heuristics and different values of $T$. These are averages over all of the corresponding problem sets. As $T$ increased, the time required increased for all heuristics and policies. The search heuristic took the most time, and using aggregation further increased the time required. This occurs because the search heuristic repeatedly evaluates the gap balance of the solutions generated, aggregating increases the densities, and evaluating the gap balance requires more effort as the number of large densities increases. The other heuristics (with or without aggregation) took much less time. For these heuristics, the time required increased when $T$ increased, but increasing $n$ made little or no difference, except for the stride scheduling heuristic, which, when $T=500$, required more time as $n$ increased. Using aggregation reduced the time required for the bottleneck heuristic for all values of $n$ and T. Using aggregation with the GR heuristic did not affect the time required. Using aggregation with the stride scheduling heuristic increased the time required slightly when $T=100$ but reduced the time required when $T=500$. For both values of $T$, using aggregation with stride scheduling heuristic required less time than the GR heuristic. 
These results show that using the stride scheduling heuristic with aggregation generates the best solutions with the least computational effort (compared to the other heuristics).

Table 8. Combinations of $T$ and $n$ used to generate instances.

\begin{tabular}{r|rrrrrrrrr}
\hline$T$ & $n$ & 10 & & & & & & & \\
\hline 100 & 10 & 20 & 30 & 40 & 50 & 60 & 70 & 80 & 90 \\
500 & 50 & 100 & 150 & 200 & 250 & 300 & 350 & 400 & 450 \\
\hline
\end{tabular}

Table 9. Average number of aggregations for the instances in each problem set.

\begin{tabular}{rrr}
$T$ & $n$ & $\begin{array}{r}\text { Average } \\
\text { number of } \\
\text { aggregations }\end{array}$ \\
\hline 100 & 10 & 2.66 \\
& 20 & 6.00 \\
& 30 & 5.71 \\
& 40 & 5.11 \\
& 50 & 4.03 \\
& 60 & 3.68 \\
& 70 & 3.23 \\
& 80 & 2.64 \\
90 & 2.07 \\
\hline 500 & 50 & 10.77 \\
100 & 9.20 \\
& 150 & 7.39 \\
200 & 6.09 \\
250 & 5.10 \\
300 & 4.34 \\
350 & 3.84 \\
400 & 3.20 \\
450 & 2.69 \\
\hline
\end{tabular}


Table 10. Average values of the count balance for the $\mathrm{H}$ and AHD solutions generated using five basic heuristics.

\begin{tabular}{|c|c|c|c|c|c|c|c|c|c|c|c|}
\hline \multirow{2}{*}{$T$} & \multirow[b]{2}{*}{$n$} & \multicolumn{2}{|c|}{ Search } & \multicolumn{2}{|c|}{ Bottleneck } & \multicolumn{2}{|c|}{ GR } & \multicolumn{2}{|c|}{ Stride 0.5} & \multicolumn{2}{|c|}{ Stride 1.0} \\
\hline & & $\mathrm{H}$ & AHD & $\mathrm{H}$ & AHD & $\mathrm{H}$ & AHD & $\mathrm{H}$ & AHD & $\mathrm{H}$ & AHD \\
\hline \multirow[t]{9}{*}{100} & 10 & 2 & 2 & 2.85 & 2 & 2.89 & 2.62 & 2.70 & 2.01 & 2.91 & 2.02 \\
\hline & 20 & 2 & 2 & 2.99 & 2 & 2.24 & 2.43 & 2.85 & 2 & 3.15 & 2 \\
\hline & 30 & 2 & 2 & 3 & 2 & 2 & 2.26 & 3 & 2 & 3.60 & 2 \\
\hline & 40 & 2 & 2 & 2.98 & 2 & 2 & 2.03 & 3.04 & 1.99 & 3.22 & 1.99 \\
\hline & 50 & 2 & 1.97 & 3 & 1.97 & 2 & 2 & 2.80 & 1.95 & 3.75 & 1.99 \\
\hline & 60 & 2 & 1.81 & 3 & 1.86 & 2 & 1.94 & 3.24 & 1.82 & 4.12 & 1.86 \\
\hline & 70 & 2 & 1.65 & 3 & 1.71 & 1.99 & 1.71 & 3.37 & 1.63 & 3.57 & 1.68 \\
\hline & 80 & 2 & 1.68 & 2.9 & 1.85 & 1.9 & 1.85 & 3.08 & 1.58 & 3.08 & 1.85 \\
\hline & 90 & 2 & 1.36 & 2.43 & 1.36 & 1.43 & 1.36 & 2.43 & 1.36 & 2.43 & 1.36 \\
\hline \multirow[t]{9}{*}{500} & 50 & 2 & 2 & 3 & 2 & 3.31 & 2.82 & 3 & 2 & 3.05 & 2 \\
\hline & 100 & 2 & 2 & 3 & 2 & 2.16 & 2.47 & 3 & 2 & 3.75 & 2 \\
\hline & 150 & 2 & 2 & 3 & 2 & 2 & 2.19 & 3.02 & 2 & 4.34 & 2 \\
\hline & 200 & 2 & 2 & 3 & 2 & 2 & 2.02 & 3.54 & 2 & 4.19 & 2 \\
\hline & 250 & 2 & 2 & 3 & 2 & 2 & 2 & 3.71 & 2 & 4.71 & 2 \\
\hline & 300 & 2 & 2 & 3 & 2 & 2 & 2 & 3.68 & 1.95 & 4.81 & 1.99 \\
\hline & 350 & 2 & 1.95 & 3 & 2 & 2 & 1.98 & 3.79 & 1.92 & 4.19 & 1.96 \\
\hline & 400 & 2 & 1.76 & 3 & 2 & 2 & 1.93 & 3.65 & 1.71 & 3.65 & 1.81 \\
\hline & 450 & 2 & 1.43 & 2.92 & 1.75 & 1.92 & 1.75 & 2.96 & 1.42 & 2.96 & 1.43 \\
\hline
\end{tabular}


Table 11. Average values of the gap balance for the $\mathrm{H}$ and AHD solutions generated using five basic heuristics.

\begin{tabular}{|c|c|c|c|c|c|c|c|c|c|c|c|}
\hline & \multirow[b]{2}{*}{$n$} & \multicolumn{2}{|c|}{ Search } & \multicolumn{2}{|c|}{ Bottleneck } & \multicolumn{2}{|c|}{ GR } & \multicolumn{2}{|c|}{ Stride 0.5} & \multicolumn{2}{|c|}{ Stride 1.0} \\
\hline & & $\mathrm{H}$ & AHD & $\mathrm{H}$ & AHD & $\mathrm{H}$ & AHD & $\mathrm{H}$ & AHD & $\mathrm{H}$ & AHD \\
\hline \multirow[t]{9}{*}{100} & 10 & 4.19 & 3.98 & 8.84 & 6.14 & 23.22 & 17.49 & 8.55 & 4.57 & 8.84 & 5.45 \\
\hline & 20 & 5.00 & 3.73 & 18.02 & 7.98 & 50.12 & 16.98 & 16.04 & 3.97 & 18.66 & 4.70 \\
\hline & 30 & 5.37 & 3.37 & 26.04 & 5.71 & 37.42 & 12.22 & 23.70 & 3.32 & 28.39 & 3.69 \\
\hline & 40 & 5.37 & 3.05 & 33.41 & 4.89 & 22.23 & 10.70 & 29.29 & 2.89 & 38.17 & 3.40 \\
\hline & 50 & 5.40 & 2.70 & 41.07 & 5.15 & 15.38 & 9.10 & 34.20 & 2.65 & 47.80 & 2.95 \\
\hline & 60 & 5.18 & 2.19 & 47.24 & 4.83 & 9.65 & 7.26 & 49.46 & 2.16 & 57.15 & 2.31 \\
\hline & 70 & 4.77 & 1.80 & 55.33 & 4.16 & 5.02 & 5.46 & 64.16 & 1.80 & 67.11 & 1.88 \\
\hline & 80 & 4.27 & 1.62 & 61.66 & 3.03 & 2.48 & 4.96 & 75.74 & 1.58 & 76.18 & 1.78 \\
\hline & 90 & 3.15 & 0.79 & 80.00 & 2.23 & 0.86 & 2.23 & 83.80 & 0.79 & 83.80 & 0.79 \\
\hline \multirow[t]{9}{*}{500} & 50 & 11.20 & 6.93 & 48.41 & 16.88 & 244.27 & 78.62 & 45.31 & 7.28 & 48.52 & 9.60 \\
\hline & 100 & 13.32 & 5.88 & 94.61 & 18.47 & 296.16 & 48.92 & 84.33 & 5.89 & 98.47 & 6.91 \\
\hline & 150 & 14.36 & 5.17 & 133.22 & 19.14 & 197.40 & 41.10 & 117.60 & 5.09 & 147.92 & 5.67 \\
\hline & 200 & 14.50 & 4.32 & 171.85 & 22.88 & 118.38 & 32.25 & 150.47 & 4.16 & 197.91 & 4.53 \\
\hline & 250 & 14.82 & 3.67 & 203.50 & 21.70 & 80.88 & 25.55 & 179.02 & 3.49 & 247.60 & 3.90 \\
\hline & 300 & 14.86 & 3.01 & 237.93 & 20.71 & 48.40 & 19.53 & 249.33 & 2.91 & 296.20 & 3.17 \\
\hline & 350 & 14.79 & 2.50 & 281.61 & 15.74 & 25.88 & 16.22 & 322.94 & 2.52 & 345.97 & 2.63 \\
\hline & 400 & 13.99 & 1.92 & 311.46 & 13.51 & 10.08 & 13.19 & 388.71 & 1.92 & 395.38 & 2.06 \\
\hline & 450 & 12.87 & 1.36 & 400.08 & 13.71 & 2.66 & 6.38 & 443.66 & 1.34 & 443.82 & 1.37 \\
\hline
\end{tabular}

Table 12. Average values of the time required to generate the $\mathrm{H}$ and AHD solutions using five basic heuristics. All values are averages over 100 instances and are in seconds.

\begin{tabular}{|c|c|c|c|c|c|c|c|c|c|c|c|}
\hline \multirow[b]{2}{*}{$T$} & \multirow[b]{2}{*}{$n$} & \multicolumn{2}{|c|}{ Search } & \multicolumn{2}{|c|}{ Bottleneck } & \multicolumn{2}{|c|}{ GR } & \multicolumn{2}{|c|}{ Stride 0.5} & \multicolumn{2}{|c|}{ Stride 1.0} \\
\hline & & $\mathrm{H}$ & $\mathrm{AHD}$ & $\mathrm{H}$ & AHD & $\mathrm{H}$ & AHD & $\mathrm{H}$ & AHD & $\mathrm{H}$ & $\mathrm{HE}$ \\
\hline \multirow[t]{9}{*}{100} & 10 & 2.9596 & 4.9964 & 0.0015 & 0.0014 & 0.0020 & 0.0023 & 0.0009 & 0.0012 & 0.0005 & 0.0012 \\
\hline & 20 & 2.1294 & 5.5540 & 0.0011 & 0.0009 & 0.0016 & 0.0019 & 0.0006 & 0.0008 & 0.0006 & 0.0008 \\
\hline & 30 & 1.8855 & 5.8556 & 0.0012 & 0.0009 & 0.0016 & 0.0019 & 0.0006 & 0.0008 & 0.0006 & 0.0008 \\
\hline & 40 & 1.7923 & 6.1272 & 0.0012 & 0.0008 & 0.0017 & 0.0019 & 0.0007 & 0.0008 & 0.0006 & 0.0008 \\
\hline & 50 & 1.7395 & 6.6604 & 0.0011 & 0.0008 & 0.0017 & 0.0019 & 0.0007 & 0.0008 & 0.0007 & 0.0008 \\
\hline & 60 & 1.6999 & 7.7378 & 0.0011 & 0.0007 & 0.0017 & 0.0018 & 0.0007 & 0.0008 & 0.0007 & 0.0008 \\
\hline & 70 & 1.6655 & 9.7657 & 0.0011 & 0.0006 & 18 & 0.0019 & 0.0008 & 0.0008 & 0.0008 & 0.0008 \\
\hline & 80 & 1.6308 & 11.1628 & 0.0011 & 0.0006 & 0.0018 & 0.0018 & 0.0008 & 0.0008 & 0.0008 & 0.0008 \\
\hline & 90 & 1.5928 & 15.1616 & 0.0010 & 0.0006 & 0.0019 & 0.0018 & 0.0009 & 0.0008 & 0.0009 & 0.0008 \\
\hline \multirow[t]{9}{*}{500} & 50 & 14.4718 & 74.3008 & 0.0135 & 0.0063 & 0.0080 & 0.0085 & 0.0035 & 0.0034 & 0.0034 & 0.0034 \\
\hline & 100 & 10.7971 & 93.3957 & 0.0175 & 0.0050 & 0.0083 & 0.0085 & 0.0046 & 0.0034 & 0.0045 & 0.0034 \\
\hline & 150 & 10.2488 & 112.3142 & 0.0192 & 0.0043 & 0.0088 & 0.0086 & 0.0057 & 0.0035 & 0.0056 & 0.0034 \\
\hline & 200 & 10.3019 & 136.1293 & 0.0207 & 0.0037 & 0.0092 & 0.0087 & 0.0068 & 0.0036 & 0.0066 & 0.0036 \\
\hline & 250 & 10.5470 & 166.3376 & 0.0215 & 0.0033 & 0.0096 & 0.0089 & 0.0079 & 0.0038 & 0.0077 & 0.0038 \\
\hline & 300 & 10.8360 & 195.6134 & 0.0217 & 0.0030 & 0.0100 & 0.0091 & 0.0090 & 0.0040 & 0.0087 & 0.0040 \\
\hline & 350 & 11.1434 & 245.6855 & 0.0220 & 0.0032 & 0.0105 & 0.0095 & 0.0101 & 0.0044 & 0.0098 & 0.0044 \\
\hline & 400 & 11.4403 & 341.7110 & 0.0219 & 0.0034 & 0.0110 & 0.0097 & 0.0111 & 0.0046 & 0.0108 & 0.0046 \\
\hline & 450 & 11.7140 & 528.2417 & 0.0205 & 0.0037 & 0.0115 & 0.0100 & 0.0122 & 0.0050 & 0.0118 & 0.0050 \\
\hline
\end{tabular}




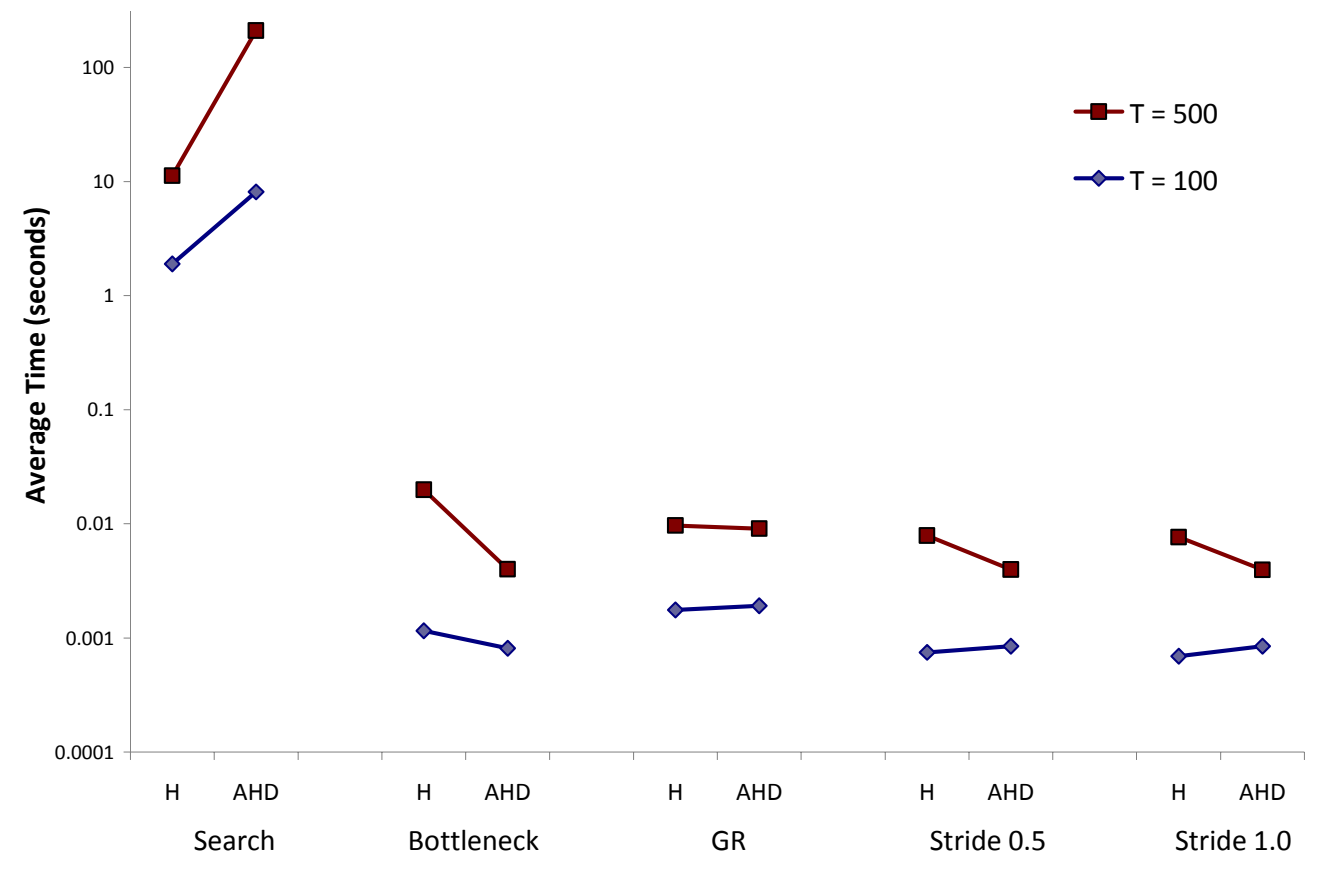

Figure 2. Average time required to generate policies for different heuristics, solutions, and values of $T$.

Times are averaged over the corresponding problem sets and instances within those sets. Note that the vertical scale is logarithmic in order to improve the clarity of the figure. 


\section{Summary and Conclusions}

This paper presented a branch-and-bound algorithm for finding optimally balanced words. Two balance measures were considered: the gap balance, which is the maximum difference in length of two factors that contain the same number of copies of a letter, and the count balance, which is the maximum difference in the number of copies of a letter for two factors of the same length.

Computational results show that instances with a medium number of letters require the most effort to solve optimally because, for each letter considered, there are many more combinations of positions that must be evaluated. Instances with many letters are easy to solve. The heuristics are able to generate many optimal solutions, and their average performance is very good.

This paper also presents an aggregation approach for the problem. We combined this approach with various heuristics in order to determine when aggregation is useful. The aggregation algorithm runs in polynomial time, but the solution generation and disaggregation algorithms require pseudo-polynomial time.

The results show that using aggregation can generate more balanced solutions. Moreover, using aggregation can reduce the computational effort needed to construct a solution.

Among the heuristics, the results of our experiments show that the GR heuristic generates balanced words without aggregation. When combined with aggregation, stride scheduling generates the best solutions. The bottleneck heuristic does not perform as well. The search algorithm of Sano et al. (2004) generates good solutions, but it requires additional computational effort.

For the BWP, we recommend using aggregation with stride scheduling. These techniques generate the best policies and require little computational effort.

The results here, along with the results of Herrmann $(2009,2010,2011)$ on using aggregation for the RTV problem and the waiting time problem, indicate that this type of aggregation approach is a powerful technique for problems that require generating a fair sequence. Unlike the previous work, which focused on specific scheduling problems, the work presented in this paper considers the more general problem of finding balanced words, which have applications in numerous domains. 
The aggregation procedure presented here cannot aggregate an instance if all of the letters have different values. For such cases, the results here indicate which heuristics perform well without aggregation. In general, it may be useful to develop and test other types of aggregation. Future work will consider systematic approaches along this line.

\section{References}

Altman, E., Gaujal, B., and Hordijk, A. (2000) Balanced sequences and optimal routing. Journal of the ACM, 47(4), 752-775.

Balinski, M.L, and Young, H.P. (1982) Fair Representation, Yale University Press, New Haven, Connecticut.

Corominas, A., Kubiak, W., and Palli, N.M. (2007) Response time variability. Journal of Scheduling, 10, $97-110$.

Hajek, B. (1985) Extremal splittings of point processes. Mathematics of Operations Research, 10, 543556.

Herrmann, J.W. (2007) Generating cyclic fair sequences using aggregation and stride scheduling. Technical Report 2007-12, Institute for Systems Research, University of Maryland, College Park. Available online at http://hdl.handle.net/1903/7082

Herrmann, J.W. (2009) Generating cyclic fair sequences for multiple servers. MISTA 2009, Dublin, Ireland, August 10-12, 2009.

Herrmann, J.W. (2010) Using aggregation to construct periodic policies for routing jobs to parallel servers with deterministic service times. Journal of Scheduling, DOI: 10.1007/s10951-010-02096.

Herrmann, J.W. (2011) Using aggregation to reduce response time variability in cyclic fair sequences. Journal of Scheduling, 14, 39-55.

Kubiak, W. (2004) Fair sequences. In Handbook of Scheduling: Algorithms, Models and Performance Analysis, Leung, J.Y-T., editor, Chapman \& Hall/CRC, Boca Raton, Florida.

Kubiak, W. (2009) Proportional Optimization and Fairness, Springer, New York. 
Nowicki, E., and Smutnicki, C. (1989) Worst-case analysis of an approximation algorithm for flow shop scheduling. Operations Research Letters, 8, 171-177.

Rock, H., and Schmidt, G. (1983) Machine aggregation heuristics in shop scheduling. Methods of Operations Research, 45, 303-314.

Rogers, D.F., Plante, R.D., Wong, R.T., and Evans, J.R. (1991) Aggregation and disaggregation techniques and methodology in optimization. Operations Research, 39(4), 553-582.

Sano, S., Miyoshi, N., and Kataoka, R. (2004) m balanced words: a generalization of balanced words. Theoretical Computer Science, 314(1-2), 97-120.

van der Laan, D.A. (2000) Routing jobs to servers with deterministic service times. Technical report 2000-20, Leiden University.

van der Laan, D. (2005) Routing jobs to servers with deterministic service times. Mathematics of Operations Research, 30(1), 195-224.

Waldspurger, C.A., and Weihl, W.E. (1995) Stride scheduling: deterministic proportional-share resource management. Technical Memorandum MIT/LCS/TM-528, MIT Laboratory for Computer Science, Cambridge, Massachusetts.

Wei, W.D., and Liu, C.L. (1983) On a periodic maintenance problem. Operations Research Letters, 2(2), 90-93. 


\title{
Online Supplement for “Finding Optimally Balanced Words for Production Planning and Maintenance Scheduling” by Jeffrey W. Herrmann
}

\author{
Jeffrey W. Herrmann \\ A. James Clark School of Engineering \\ University of Maryland \\ College Park, MD 20742 \\ jwh2@umd.edu
}

\section{Part A. Algorithms for the Heuristics.}

\section{Count balance algorithm}

The count balance algorithm determines the count balance of the infinite word generate from a finite word and can be described as follows. The input is an instance $\left(x_{1}, \ldots, x_{n}\right)$ with $x_{1} \geq x_{2} \geq \cdots \geq x_{n}$. Let $T=x_{1}+\cdots+x_{n}$. In this algorithm, $S_{t}$ refers to the letter in position $t$ of $S$. Note that, if $n=1$, then the count balance of $S$ equals 0 .

1. Set $c=1$. Set $L_{i}=\max _{t=1, \ldots, T}\left\{t: S_{t}=i\right\}$ and $g_{i}=0$ for all $i=1, \ldots, n$.

2. For $t=1, \ldots, T$, perform the following steps:

a. Let $i=S_{t}$. Increase $g_{i}$ by 1 . If $L_{i}<t$, set $\Delta_{i g_{i}}=t-L_{i}-1$; else set

$$
\Delta_{i g_{i}}=T+t-L_{i}-1
$$

3. For $i=1, \ldots, n$, perform the following steps:

$a$. If $x_{i} \geq c+1$ and $\min _{j=1, \ldots, x_{i}}\left\{\Delta_{i j}\right\}<\max _{j=1, \ldots, x_{i}}\left\{\Delta_{i j}\right\}$, go to step $b$. Else, go to next $i$.

$b$. For $j=1, \ldots, x_{i}$, perform the following step:

i. Set $M_{j}^{A}=\max _{k=0, \ldots, x_{i}-1}\left\{\sum_{q=1}^{j} \Delta_{i, k+q}\right\}+j-1 \quad$ and $\quad M_{j}^{B}=\min _{k=0, \ldots, x_{i}-1}\left\{\sum_{q=1}^{j} \Delta_{i, k+q}\right\}+j+1$

(in these summations, $\Delta_{i, k+q}=\Delta_{i, k+q-x_{i}}$ if $k+q>x_{i}$ ). 
c. Set $p=1$.

d. If $M_{p}^{A} \geq M_{p+c-1}^{B}$, increase $c$ by 1 and go back to step $c$.

$e$. Increase $p$ by 1 . If $p \leq x_{i}-c+1$, then go back to step $d$.

4. Return $c$ as the count balance.

In step 3.d., if $M_{p}^{A} \geq M_{p+c-1}^{B}$, then there is an integer $m$, with $M_{p}^{A} \geq m \geq M_{p+c-1}^{B}$, such that there is a factor $x$ of length $m$ with at most $p-1$ copies of $i$ (because $M_{p}^{A}$ is the length of the longest factor with only $p-1$ copies of $i$ ) and a factor $y$ of length $m$ with at least $p+c$ copies of $i$ (because $M_{p+c-1}^{B}$ is the length of the shortest factor with $p+c$ copies of $i$ ). Thus, $|y|_{i}-|x|_{i} \geq p+c-(p-1)=c+1$, so the count balance must be at least $c+1$.

\section{Gap balance algorithm}

The gap balance algorithm determines the gap balance of the infinite word generate from a finite word and can be described as follows. The input is an instance $\left(x_{1}, \ldots, x_{n}\right)$ with $x_{1} \geq x_{2} \geq \cdots \geq x_{n}$. Let $T=x_{1}+\cdots+x_{n}$. In this algorithm, $S_{t}$ refers to the letter in position $t$ of $S$. Note that, if $n=1$, then the count balance of $S$ equals 0 .

To evaluate the gap balance, we need the smallest value $v$ such that $\left|W^{\prime}\right|=|W|+v+1$ satisfies $\left|W^{\prime}\right|_{i} \geq|W|_{i}+1$. Equivalently, $\left|W^{\prime}\right|_{i} \leq|W|_{i}$ implies $\left|W^{\prime}\right| \leq|W|+v$, which is the same as $v \geq\left|W^{\prime}\right|-|W|$. So, for the letter $i$, we need to look for the shortest factor between two copies of $i$ and the longest factor so that both have the same number of copies of $i$. We define a "gap" as a factor that occurs between two copies of the letter $i$. There are exactly $x_{i}$ gaps for letter $i$. The non-negative length of a gap $\Delta_{i k}$ is the number of positions between the copies of $i$. The shortest factor between two copies of $i$ that has $j$ copies of $i$ and the longest factor that has $j$ copies of $i$ will be some $j+1$ consecutive gaps plus the $j$ copies of $i$. In the gap balance algorithm, $\delta_{j}$ (computed in step 3.b) is the difference between the lengths of the shortest and longest factors that have $j$ copies of $i$. 
1. Set $c=1$. Set $L_{i}=\max _{t=1, \ldots, T}\left\{t: S_{t}=i\right\}$ and $g_{i}=0$ for all $i=1, \ldots, n$.

2. For $t=1, \ldots, T$, perform the following steps:

a. Let $i=S_{t}$. Increase $g_{i}$ by 1 . If $L_{i}<t$, set $\Delta_{i g_{i}}=t-L_{i}-1$; else set

$$
\Delta_{i g_{i}}=T+t-L_{i}-1
$$

3. For $i=1, \ldots, n$, perform the following steps:

a. If $x_{i} \geq 2$ and $\min _{j=1, \ldots, x_{i}}\left\{\Delta_{i j}\right\}<\max _{j=1, \ldots, x_{i}}\left\{\Delta_{i j}\right\}$, go to step $b$.

Else, set $b_{i}=0$ and go to next $i$.

$b$. For $j=0, \ldots, x_{i}-2$, set $\delta_{j}=\max _{k=1, \ldots, x_{i}}\left\{\sum_{q=0}^{j} \Delta_{i, k+q}\right\}-\min _{k=1, \ldots, x_{i}}\left\{\sum_{q=0}^{j} \Delta_{i, k+q}\right\}$

(in these summations, $\Delta_{i, k+q}=\Delta_{i, k+q-x_{i}}$ if $k+q>x_{i}$ ).

$c$. Set $b_{i}=\max _{j=0, \ldots, x_{i}-2}\left\{\delta_{j}\right\}$.

4. Return $m=\max _{i=1, \ldots, n}\left\{b_{i}\right\}$ as the gap balance of $S$.

\section{GR algorithm}

The GR algorithm can be described as follows. The input is an instance $\left(x_{1}, \ldots, x_{n}\right)$ with $x_{1} \geq x_{2} \geq \cdots \geq x_{n}$. Let $T=x_{1}+\cdots+x_{n}$.

1. Set $X_{i}=\sum_{k=i}^{n} x_{k}, N_{i}=0$, and $R_{i}=0$ for all $i=1, \ldots, n$.

2. For $t=0, \ldots, T-1$, perform the following steps:

a. Set $\Delta_{i}=x_{i}\left(1+R_{i}\right)-N_{i} X_{i}$ for all $i=1, \ldots, n$. 
$b$. Set $P^{t}$ to the letter $s$ where $\left.s=\min \left\{i: \Delta_{i}>0\right\}.\right)$

c. Increase $N_{s}$ by 1.

$d$. Increase $R_{i}$ by 1 for all $i=1, \ldots, s$.

3. Return $P^{0}, \ldots, P^{T-1}$ as the solution.

Table A.1. The construction of a periodic solution for the instance $(4,3,2)$ using the GR heuristic.

\begin{tabular}{c|ccccccccc|}
\cline { 2 - 9 }$t$ & 0 & 1 & 2 & 3 & 4 & 5 & 6 & 7 & 8 \\
\hline$N_{1}$ & 0 & 1 & 1 & 2 & 2 & 3 & 3 & 4 & 4 \\
$N_{2}$ & 0 & 0 & 1 & 1 & 2 & 2 & 2 & 2 & 3 \\
$N_{3}$ & 0 & 0 & 0 & 0 & 0 & 0 & 1 & 1 & 1 \\
\hline$R_{1}$ & 0 & 1 & 2 & 3 & 4 & 5 & 6 & 7 & 8 \\
$R_{2}$ & 0 & 0 & 1 & 1 & 2 & 2 & 3 & 3 & 4 \\
$R_{3}$ & 0 & 0 & 0 & 0 & 0 & 0 & 1 & 1 & 1 \\
\hline$\Delta_{1}$ & 4 & -1 & 3 & -2 & 2 & -3 & 1 & -4 & 0 \\
$\Delta_{2}$ & 3 & 3 & 1 & 1 & -1 & -1 & 2 & 2 & 0 \\
$\Delta_{3}$ & 2 & 2 & 2 & 2 & 2 & 2 & 2 & 2 & 2 \\
\hline$P^{t}$ & 1 & 2 & 1 & 2 & 1 & 3 & 1 & 2 & 3 \\
\hline
\end{tabular}

\section{Parameterized stride scheduling algorithm}

The parameterized stride scheduling algorithm can be described as follows. The inputs are an instance $\left(x_{1}, \ldots, x_{n}\right)$ and the parameter $\delta$. Let $T=x_{1}+\cdots+x_{n}$.

1. Initialization. $N_{i}=0$ for $i=1, \ldots, n$.

2. For $t=0, \ldots, T$-1, perform the following steps:

$a$. Set $P^{t}$ to the letter $s$ that has the largest value of $\frac{x_{i}}{N_{i}+\delta}$. In case of a tie, select the letter with smallest $x_{i}$.

b. Increase $N_{s}$ by 1 . 
3. Return $P^{0}, \ldots, P^{T-1}$ as the solution.

Table A.2. The construction of a solution for the instance $(4,3,2)$ using the stride scheduling heuristic with $\delta=0.5$.

\begin{tabular}{c|ccccccccc|}
\cline { 2 - 9 }$t$ & 0 & 1 & 2 & 3 & 4 & 5 & 6 & 7 & 8 \\
\hline$N_{1}$ & 0 & 1 & 1 & 2 & 2 & 3 & 3 & 4 & 4 \\
$N_{2}$ & 0 & 0 & 1 & 1 & 2 & 2 & 2 & 2 & 3 \\
$N_{3}$ & 0 & 0 & 0 & 0 & 0 & 0 & 1 & 1 & 1 \\
\hline$x_{1} /\left(N_{1}+\delta\right)$ & 8 & 2.67 & 2.67 & 2.67 & 1.6 & 1.6 & 1.14 & 1.14 & 1.14 \\
$x_{2} /\left(N_{2}+\delta\right)$ & 6 & 6 & 2 & 2 & 2 & 1.2 & 1.2 & 1.2 & 0.86 \\
$x_{2} /\left(N_{2}+\delta\right)$ & 4 & 4 & 4 & 1.33 & 1.33 & 1.33 & 1.33 & 0.8 & 0.8 \\
\hline$P^{t}$ & 1 & 2 & 3 & 1 & 2 & 1 & 3 & 2 & 1 \\
\hline
\end{tabular}

\section{Bottleneck algorithm}

The bottleneck algorithm can be described as follows. The input is an instance $\left(x_{1}, \ldots, x_{n}\right)$. Let $T=x_{1}+\cdots+x_{n}$.

1. Set $w=0$ and $y_{i}=1$ for all $i=1, \ldots, n$.

2. For $i=1, \ldots, n$ and $j=1, \ldots, x_{i}$, calculate the following quantities:

$$
\begin{gathered}
E S T_{i j}=\left\lceil\frac{T(j-1)+w}{x_{i}}-1\right\rceil \\
L S T_{i j}=\left\lfloor\frac{T j-w}{x_{i}}\right\rfloor
\end{gathered}
$$

3. For $k=0, \ldots, T-1$, perform the following steps:

$a$. Let $R=\left\{i: y_{i} \leq x_{i}, E S T_{i y_{i}} \leq k, L S T_{i y_{i}} \geq k\right\}$. If $R$ is empty, go to Step 5 .

$b$. Let $i$ be the product in $R$ that has the smallest $L S T_{i y_{i}}$.

c. Assign product $i$ to position $k+1$, and increase $y_{i}$ by 1 . 
4. Save the current sequence. If $w<\max \left\{x_{i}\right\}$, then set $w$ to the value of the smallest $x_{i}$ that is greater than $w$, and go to Step 2. Otherwise, go to Step 5 .

5. Return the last saved sequence.

\section{Search algorithm}

The Search algorithm can be described as follows. The input is an instance $\left(x_{1}, \ldots, x_{n}\right)$ with $x_{1} \geq x_{2} \geq \cdots \geq x_{n}$. Let $T=x_{1}+\cdots+x_{n}$. Let $M$ be the total number of samples.

1. For $a=1, \ldots, M$, perform the following steps:

$a$. Randomly select $\phi_{i} \in\left[0, T / x_{i}\right]$ for all $i=1, \ldots, n$.

$b$. For $t=1, \ldots, T$, set $P^{t}$ to the letter $i$ with $\phi_{i}=\min \left\{\phi_{1}, \ldots, \phi_{n}\right\}$ and then increase $\phi_{i}$ by $T / x_{i}$.

c. Determine the gap balance of $P$. If this is the best gap balance so far, save $P$.

2. Return the best word found. 


\section{Part B. Aggregation and Disaggregation Algorithms.}

The notation used in the algorithm that follows enables us to keep track of the aggregations in order to describe the disaggregation of a sequence precisely. Let $I_{0}$ be the original instance (alphabet) and $I_{k}$ be the $k$-th instance generated from $I_{0}$. Let $n_{k}$ be the number of letters in instance $I_{k}$. Let $B_{j}$ be the set of letters that form the new letter $j$, and let $B_{j}(i)$ be the $i$-th letter in that set. As the aggregation algorithm is presented, we describe its operation on the following five-letter example: $I_{0}=(3,2,2,1,1), n=5$, and $T=9$.

Aggregation. Given: an instance $I_{0}$ with values $\left(x_{1}, x_{2}, \ldots, x_{n}\right)$.

1. Initialization. Let $k=0$ and $n_{0}=n$.

2. Stopping rule. If all of the letters in $I_{k}$ have different values, return $I_{k}$ and $H$ $=k$ because no further aggregation is possible. Otherwise, let $G$ be the set of letters with the same value such that any smaller value is unique.

Example. With $k=0, G=\{4,5\}$ because $x_{4}=x_{5}$.

3. Aggregation. Let $m=|G|$ and let $i$ be one of the letters in $G$. Create a new letter $n+k+1$ with value $x_{n+k+1}=m x_{i}$. Create the new instance $I_{k+1}$ by removing from $I_{k}$ all $m$ letters in $G$ and adding letter $n+k+1$. Set $B_{n+k+1}=G . n_{k}=n_{k-1}-m+1$. Increase $k$ by 1 and go to Step 2 .

Example. With $k=0$ and $G=\{4,5\}$, the new letter 6 has value $x_{6}=2 \times 1=2 . B_{6}=\{4,5\}$.

The letters in $I_{1}$ are $\{1,2,3,6\}$. When $k=1, G=\{2,3,6\}$. The new letter 7 has value $x_{7}=3 \times 2=6$, and $B_{7}=\{2,3,6\}$. The letters in $I_{2}$ are $\{1,7\}$, which have different values. Table B.1 describes the instances created for this example. 
Table B.1. The values for the five original letters in the example instance $I_{0}$ and the two new letters in the aggregate instances $I_{1}$ and $I_{2}$.

\begin{tabular}{|c|ccccc|cc|}
\cline { 2 - 7 } \multicolumn{1}{c|}{} & $x_{1}$ & $x_{2}$ & $x_{3}$ & $x_{4}$ & $x_{5}$ & $x_{6}$ & $x_{7}$ \\
\hline$I_{0}$ & 3 & 2 & 2 & 1 & 1 & & \\
$I_{1}$ & 3 & 2 & 2 & & & 2 & \\
$I_{2}$ & 3 & & & & & & 6 \\
\hline
\end{tabular}

In the following algorithm, $j=S_{k}(a)$ means that letter $j$ is in position $a$ in solution $S_{k}$, and $B_{n+k}(i)$ is the $i$-th letter in $B_{n+k}$.

Disaggregation. Given: The instances $I_{0}, \ldots, I_{H}$ and the solution $S_{H}$, a feasible solution for the instance $I_{H}$.

1. Initialization. Let $k=H$.

2. Set $m=\left|B_{n+k}\right|$ and $i=1$.

3. For $a=0, \ldots, T-1$, perform the following step:

a. If $S_{k}(a)<n+k$, assign $S_{k-1}(a)=S_{k}(a)$. Otherwise, assign $S_{k-1}(a)=B_{n+k}(i)$, increase $i$ by 1 , and, if $i>m$, set $i=1$.

4. Decrease $k$ by 1 . If $k>0$, go to Step 2. Otherwise, stop and return $S_{0}$.

Example. Consider the aggregation of the instance (3, 2, 2, 1, 1) presented earlier and the solution $S_{2}=7-7-1-7-7-1-7-7-1$, which is a feasible solution for the aggregated instance $I_{2}$. When $k=2, n+k=7$, and $B_{7}=\{2,3,6\}$. The positions in $S_{2}$ that are assigned to letter 7 will be reassigned to letters 2,3 , and 6 . The resulting solution $S_{1}=2-3-1-6-2-1-3-6-1$. 
When $k=1, n+k=6$, and $B_{6}=\{4,5\}$. The positions in $S_{1}$ that are assigned to letter 6 will be reassigned to letters 4 and 5. The resulting solution $S_{0}=2-3-1-4-2-1-3-5-1$. Table B.2 lists these three solutions.

Table B.2. The disaggregation of solution $S_{2}$ for instance $I_{2}$ in the example. The first row is $S_{2}$, a feasible solution for instance $I_{2}$. The second row is $S_{1}$, a feasible solution for instance $I_{1}$. The third row is $S_{0}$, a feasible solution for instance $I_{0}$.

\begin{tabular}{c|ccccccccc}
\cline { 2 - 9 }$a$ & 0 & 1 & 2 & 3 & 4 & 5 & 6 & 7 & 8 \\
\hline$S_{2}(a)$ & 7 & 7 & 1 & 7 & 7 & 1 & 7 & 7 & 1 \\
$S_{1}(a)$ & 2 & 3 & 1 & 6 & 2 & 1 & 3 & 6 & 1 \\
$S_{0}(a)$ & 2 & 3 & 1 & 4 & 2 & 1 & 3 & 5 & 1 \\
\hline
\end{tabular}




\section{Part C. Proofs.}

Theorem 1. Disaggregating a word that has a positive count balance does not increase its count balance.

Proof. Consider the aggregated word $U+$ and the disaggregated word $U-$. The disaggregation replaces $m x$ copies of the letter $j$ in $U+$ by $x$ copies of the $m$ letters in $B_{j}$ in a round-robin manner.

Let $c$ be the count balance of $U+$ and let $y$ and $z$ be factors of $U$ such that $c=|z|_{j}-|y|_{j}$.

Let $a$ be one of the letters that replaces letter $j$. Because the copies of $j$ are replaced in a roundrobin manner, $|y|_{a}=\left\lfloor|y|_{j} / m\right\rfloor$ or $|y|_{a}=\left\lceil|y|_{j} / m\right\rceil$. Likewise, $|z|_{a}=\left\lfloor|z|_{j} / m\right\rfloor$ or $|z|_{a}=\left\lceil|z|_{j} / m\right\rceil$. Therefore, $|z|_{a}-|y|_{a} \leq\left\lceil|z|_{j} / m\right\rceil-\left\lfloor|y|_{j} / m\right\rfloor$

If $c=1$, we consider three cases. First, if $|z|_{j} \equiv 0(\bmod m)$, then $|z|_{a}=|z|_{j} / m$ and $\left\lfloor|y|_{j} / m\right\rfloor=|z|_{j} / m-1$. Thus, $|z|_{a}-|y|_{a} \leq 1$. Second, if $|y|_{j} \equiv 0(\bmod m)$, then $|y|_{a}=|y|_{j} / m$ and $\left\lceil|z|_{j} / m\right\rceil=|y|_{j} / m+1$. Thus, $|z|_{a}-|y|_{a} \leq 1$. Otherwise, $\left.\left\lceil|z|_{j} / m\right\rceil=\left.|| y\right|_{j} / m\right\rfloor+1$, so $|z|_{a}-|y|_{a} \leq 1$.

If $c=2$, we consider the following three cases. First, if $|y|_{j} \equiv 0(\bmod m)$, then $|y|_{a}=|y|_{j} / m$ and $\left[|z|_{j} / m\right\rceil=|y|_{j} / m+1$. Thus, $|z|_{a}-|y|_{a} \leq 1$. Second, if $|y|_{j} \equiv m-1(\bmod m)$, then $|z|_{j} \equiv 1(\bmod m)$ and $\left\lceil|z|_{j} / m\right\rceil=\left\lfloor|y|_{j} / m\right\rfloor+2$. Therefore, $|z|_{a}-|y|_{a} \leq 2 . \quad$ Otherwise, $\left\lceil|z|_{j} / m\right\rceil=\left\lfloor|y|_{j} / m\right\rfloor+1$, so $|z|_{a}-|y|_{a} \leq 1$ 
If $c \geq 3$, then we note that $\left[|z|_{j} / m\right\rceil<|z|_{j} / m+1$ and $\left.\left.|| y\right|_{j} / m\right]>|y|_{j} / m-1$. Therefore, $|z|_{a}-|y|_{a} \leq\left(|z|_{a}-|y|_{a}\right) / m+2=c / m+2 \leq c / 2+2$. This difference must be an integer, so, when $c=3,|z|_{a}-|y|_{a} \leq 3$. For $c \geq 4$, it is clear than $c / 2+2 \leq c$.

These cases all show that, in the disaggregated word, $|z|_{a}-|y|_{a} \leq c$, so the count balance of the disaggregated word is not larger than the count balance of the aggregated word. Q.E.D.

Theorem 2. Disaggregating a word does not increase its gap balance.

Proof. Consider the aggregated word $U+$ and the disaggregated word $U-$. The disaggregation replaces $m x$ copies of the letter $j$ in $U+$ by $x$ copies of the $m$ letters in $B_{j}$ in a round-robin manner.

Let $v$ be the gap balance of a letter $i$ in $U$ - that replaced the letter $j$ in $U+$. Then there exist factors $W^{\prime}$ and $W$ such that $\left|W^{\prime}\right|_{i}=|W|_{i}$ and $\left|W^{\prime}\right|=|W|+v$. Moreover, the positions immediately before and after $W^{\prime}$ and $W$ contain the letter $i$. Let $t=\left|W^{\prime}\right|_{i}=|W|_{i}$. Therefore, factors $W^{\prime}$ and $W$ contain $t$ copies of $i$ and $t+1$ copies of each of the $m-1$ other letters than replaced the letter $j$. Therefore, in the word $U+$, the positions corresponding to $W^{\prime}$ contain $t+(m-1)(t+1)$ copies of $j$. Likewise, the positions corresponding to $W$ contain $t+(m-1)(t+1)$ copies of $j$. Moreover, the positions immediately before and after $W^{\prime}$ and $W$ contain the letter $j$. Because $\left|W^{\prime}\right|=|W|+v$, the gap balance of the letter $j$ in $U+$ must be at least $v$. Therefore, the gap balance of any letter that replaced $j$ is less than or equal to the gap balance of $j$. The gap balance of no other letter changes because of the disaggregation, so the gap balance of the disaggregated word is less than or equal to the gap balance of the aggregated word. Q.E.D. 